\title{
Self-similar pattern formation and continuous mechanics of self-similar systems
}

\author{
A. V. Dyskin \\ School of Civil and Resource Engineering, The University of Western Australia, Australia \\ Received: 9 February 2006 - Published in Hydrol. Earth Syst. Sci. Discuss.: 25 July 2006 \\ Revised: 1 December 2006 - Accepted: 1 December 2006 - Published: 17 January 2007
}

\begin{abstract}
In many cases, the critical state of systems that reached the threshold is characterised by self-similar pattern formation. We produce an example of pattern formation of this kind - formation of self-similar distribution of interacting fractures. Their formation starts with the crack growth due to the action of stress fluctuations. It is shown that even when the fluctuations have zero average the cracks generated by them could grow far beyond the scale of stress fluctuations. Further development of the fracture system is controlled by crack interaction leading to the emergence of self-similar crack distributions. As a result, the medium with fractures becomes discontinuous at any scale. We develop a continuum fractal mechanics to model its physical behaviour. We introduce a continuous sequence of continua of increasing scales covering this range of scales. The continuum of each scale is specified by the representative averaging volume elements of the corresponding size. These elements determine the resolution of the continuum. Each continuum hides the cracks of scales smaller than the volume element size while larger fractures are modelled explicitly. Using the developed formalism we investigate the stability of selfsimilar crack distributions with respect to crack growth and show that while the self-similar distribution of isotropically oriented cracks is stable, the distribution of parallel cracks is not. For the isotropically oriented cracks scaling of permeability is determined. For permeable materials (rocks) with self-similar crack distributions permeability scales as cube of crack radius. This property could be used for detecting this specific mechanism of formation of self-similar crack distributions.
\end{abstract}

Correspondence to: A. V. Dyskin

(adyskin@cyllene.uwa.edu.au)

\section{Introduction}

Fluid flow through rocks and the Earth's crust is at large extent controlled by existing fracture systems and networks. These systems often have complex geometry with strong showing of self-similarity and fractality (e.g., Scholz and Aviles, 1986; Gelikman and Pisarenko, 1989; Scholz, 1990; Olding, 1992; Barton and Zoback, 1992; Turcotte, 1993; Gillespie et al., 1993; Yamamoto et al., 1993; Dubois, 1998; Hodkiewicz et al., 2005). Hence, understanding of the flaw mechanisms requires the understanding of the formation and evolution of fracture systems resulted from a combined action of loading acting upon and interaction between the fractures.

One of the features of complex systems is selforganisation and formation of specific patterns in their structure or behaviour which have no resemblance with the original structure of the system or behaviour of its components. Pattern formation of observed in many natural and artificial systems (e.g., Walgraef, 1997), in particular, in such processes as crystal growth and other growth phenomena (e.g., Langer, 1980, 1989), dislocation movement (e.g., Weiss and Marsan, 2003), movement of granular materials (e.g., Baxter et al., 1989). The pattern formation is usually related to the situations when a system parameter or state variable reaches a certain threshold value.

In many cases a prerequisite for pattern formation is the presence of non-linearity in one form or another. An important feature of non-linear systems is that they do not support the superposition principle. This is often considered as a nuisance as it causes additional complications to the modelling; however there is more than that. If the input signal is in a form of noise with zero mean, then the linear system $(L)$ will necessarily produce the output with zero mean, while some non-linear systems $(N L)$ react by delivering non-zero shift to the output:

$$
\langle u\rangle=0 \Rightarrow\langle L u\rangle=0, \quad\langle u\rangle=0 \Rightarrow\langle N L u\rangle \neq 0
$$

Published by Copernicus GmbH on behalf of the European Geosciences Union. 
Here the symbol $\langle$.$\rangle stands for the operator of averaging.$ This statement albeit obvious suggests that in some nonlinear systems trivial noise may have non-trivial implications. In particular, by this mechanism a fracture system could evolve even if the total applied force is zero (Dyskin, 1999, 2002). In this case the role of the input noise is played by spatial stress fluctuations (spatial stress non-uniformity).

Fracture formation is a non-linear phenomenon due to its threshold nature and irreversibility. In classical scenarios fracturing starts when the load reaches a threshold. When propagation of a single crack is restricted, new cracks appear and form a certain pattern as dictated by the loading conditions and crack interactions. In the case of spatial stress fluctuations with zero mean the role of the loading parameter is played by the amplitude (or standard deviation) of the fluctuations: local fractures appear when the amplitude of stress non-uniformity reaches a critical value. More precisely, the local fracturing occurs when and where the magnitude of a corresponding tensile stress component exceeds local strength. Since the probability of this happening, increases with the increase in the number of loci subjected to this model of stress fluctuations and eventually upon the size of the part of the material in question, a size effect in strength can be expected. This mechanism is investigated in Sect. 2 of this paper.

Types of the pattern formed in complex systems are numerous ranging from simple periodic ones to extremely complex patterns typical for living matter. Amongst spatial patterns there is however one specific type of patterns outstanding in its ubiquity - the self-similar patterns whose parameters or state variables are expressible via power functions of scale. The self-organised criticality (Bak et al., 1987) is a well known, but not the only, mechanism of producing selfsimilar patterns. Self-similarity, i.e. the absence of a characteristic size is also typical for physical systems at critical stage or at phase transition (e.g., Haken, 1978), in percolation phenomena (e.g., Stauffer and Aharony, 1992) or in other situations when interaction between the elements of the system create patterns which are independent of the elements (and hence the size of the elements) and do not involve emergence of intermediate characteristic sizes. Power law distributions also provide approximations for a range of complex natural phenomena (e.g. Sornette, 2000) and thus provide the cases for apparent self-similarity.

One of the mechanisms of producing self-similar patterns is the interaction between the elements of the system. If the elements are different (say have different sizes) or nonregularly located, the interaction can magnify the influence of same elements at the expense of the other. For example in the percolation models where the connectedness of marked elements is considered, the interaction is essentially geometrical: if marked elements are situated close enough they form clusters. The latter have larger surface area than single marked elements and thus can easier be connected to other marked elements or clusters thereof. The case of cracks in the condition of stable growth delivers anther example. Larger cracks are, on average, more susceptible to the influence of interaction than the small ones. Thus the large cracks are capable of growing to larger extent. We discuss this mechanism in Sect. 3 of this paper.

Section 4 introduces the method of modelling multiscale self-similar crack distributions using the notions of continuum mechanics. Section 5 discusses stability of self-similar crack distributions with respect to crack growth. Section 6 considers transport properties such as permeability of materials with self-similar crack distributions.

\section{Non-linearity and fluctuations: a mechanism of cracking in the absence of applied force}

In what follows we assume that the material is isotropic and elastic such that all non-linear effects are solely related to the formation and propagation of fractures or cracks. The formation of fracture systems is usually attributed to two major factors: the application of external loading or the action of residual stresses "frozen" in the material. Appearance of the latter is not directly related to the applied load but rather caused by shrinkage or other non-uniform deformation of the material due to cooling or phase transition. Superficially these two cases look completely different: the residual stresses form a non-uniform stress field with vanishing average (as there is no external load applied), while the external load directly induces stresses which after reaching the strength limit produce fracturing. It is important to emphasise, that due to heterogeneity of the material the stresses created by the loading are not uniform and usually could be split into two parts: a uniform or slightly varying part and a varying part with vanishing average. Thus the stress field $\sigma_{i j}(\boldsymbol{x})$, where $\boldsymbol{x}$ is a point in the material, can be represented as a sum of a slowly varying (or homogeneous) part $\sigma_{i j}^{0}$ and fluctuations $\Delta \sigma_{i j}(\boldsymbol{x})$ with zero mean:

$\sigma_{i j}(\boldsymbol{x})=\sigma_{i j}^{0}+\Delta \sigma_{i j}(\boldsymbol{x}), \quad\left\langle\Delta \sigma_{i j}\right\rangle=0$

When $\sigma_{i j}^{0}=0$, the total force of the stress distribution being proportional to the average stress vanishes. Had the system be linear this would mean that the stress fluctuations have no effect on the fracture process. However, the fracture process is highly non-linear. In particular, in brittle materials even the sense of the stresses matter as only positive (tensile) stress can directly induce fracturing, while the compressive stress should do it through local transition to tensile stress (that is why in brittle materials compressive strength is an order of magnitude higher than the tensile one). Therefore, fractures are preferentially formed in the areas subjected to stress fluctuations with positive sign.

In the case of residual stresses, obviously, $\sigma_{i j}^{0}=0$, so only tensile parts of stress fluctuations, i.e. the loci where the corresponding component of stress fluctuations is positive, 


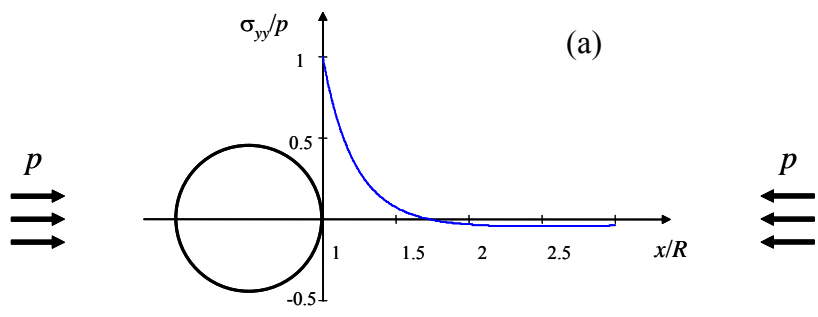

(b)

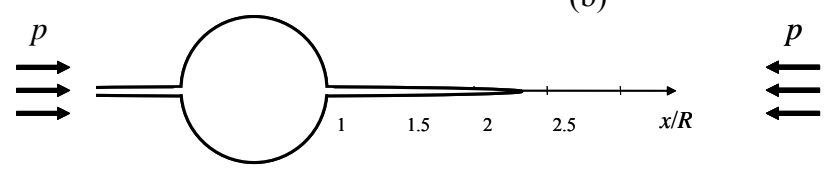

Fig. 1. Stress disturbance and subsequent cracking produced by a pore in uniaxial compression: (a) distribution of the normal stress component $\sigma_{y y}$; (b) crack starts at the pore contour by $\sigma_{y y}$ and then grows to some extend further.

should be considered as a part of the mechanism of fracture formation. In the case of applied load there are situations when tensile components of $\sigma_{i j}^{0}$ also vanish such that, as far as the crack formation is concerned, again only the tensile parts of stress fluctuations matter. The main example is failure in uniaxial compression, Fig. 1a. In this case cracks can only form in the directions parallel to the load direction where no force is applied from outside. Local tensile stress appear as a part of stress non-uniformity caused by the material heterogeneity, for instance by the presence of defects (e.g., pores as in Fig. 1a).

The presence of defects or other heterogeneities alters the stress field without changing the total force and in same cases transfer compressive stress into tensile. Figure 1a illustrates one of the mechanisms of such a transition. A circular pore of radius $R$ loaded by compressive stress of magnitude $p$ in $\mathrm{x}$-direction induces vertical normal stress $\sigma_{y y}$ on the x-axis, which would be absent in the absence of the hole. The distribution shown in Fig. 1 (due to symmetry only the case $x>0$ is shown) is of a fluctuational nature with zero mean. The stress near the hole is positive (tensile) which when reaches sufficient magnitude, gives rise to tensile cracks, Fig. $1 \mathrm{~b}$.

An important observation should be made from this example: the crack is generated at the place of maximum stress (at the pore contour in this case) and then can grow beyond the area of the action of tensile stress. In general, this point is illustrated by Fig. 2, where a possible distribution of stress fluctuations is sketched. The crack is formed at the place where the tensile stress reaches its maximum (the origin of the coordinate set is placed there). Then, the crack grows to the size considerably exceeding the characteristic length of stress fluctuations.

In order to analyse this situation we consider a 2-D case of a planar crack of length $2 a$, Fig. 2 subjected to the stress

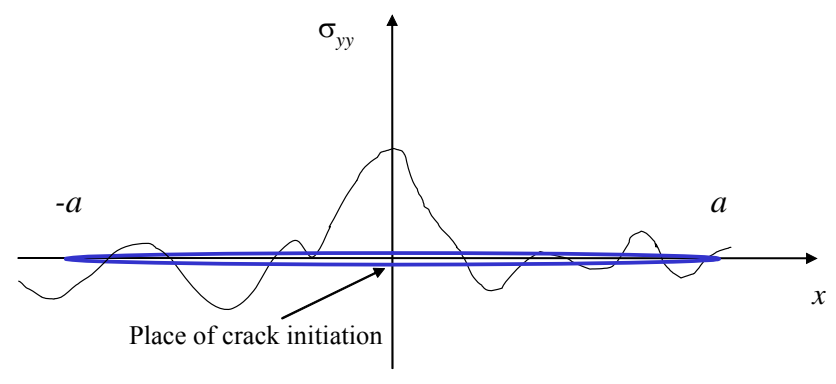

Fig. 2. Crack growth caused by stress fluctuations.

fluctuations $\Delta \sigma_{y y}(x)$ having zero average. We will assume that the stress fluctuations are represented by an ergodic random process such that statistical average coincides with the spatial average:

$\overline{\Delta \sigma_{y y}}(x)=\left\langle\Delta \sigma_{y y}\right\rangle=0$

Consider a realisation $\Delta \sigma_{y y}(x)$ of the random field of stress fluctuations. Suppose that the crack is placed in the material at the locus where this stress acts. We also assume, for the sake of simplicity, that the emerging crack in not infinitesimal thin such that the compressive parts of the stress fluctuations (negative parts of Fig. 2) are insufficient to bring the opposite faces of the crack to contact. The elastic solution for this case shows that the stress at the crack tip becomes infinite, increasing as inverse square root of the distance to the crack tip (so-called the square root singularity). This prevents one from comparing the stress at the crack tip with the local strength to decide whether the crack is going to grow or not.

In reality there can be no infinite stresses of course, just the zone near the crack tip is no longer elastic and hence the elastic solution is no longer applicable. The Linear Elastic Fracture Mechanics bypasses this problem by simply using the coefficient at the square root singularity, so-called the stress intensity factor in the criterion of crack growth as follows (e.g., Tada et al., 1985):

$K_{I}=K_{I c}, \quad K_{I}=\frac{1}{\sqrt{\pi a}} \int_{-a}^{a} \Delta \sigma_{y y}(t) \sqrt{\frac{a+t}{a-t}} d t$

where $K_{I}$ is the mode I stress intensity factor, which is a coefficient at the square root singularity of the stress field near the crack tip, $K_{I c}$ is the fracture toughness - the critical value of the stress intensity factor at which the crack starts growing.

As the crack location in this example is independent of the stress field, the average stress intensity factor is

$\bar{K}_{I}=\frac{1}{\sqrt{\pi a}} \int_{-a}^{a} \overline{\Delta \sigma_{y y}}(t) \sqrt{\frac{a+t}{a-t}} d t=0$,

as property (3) dictates. 


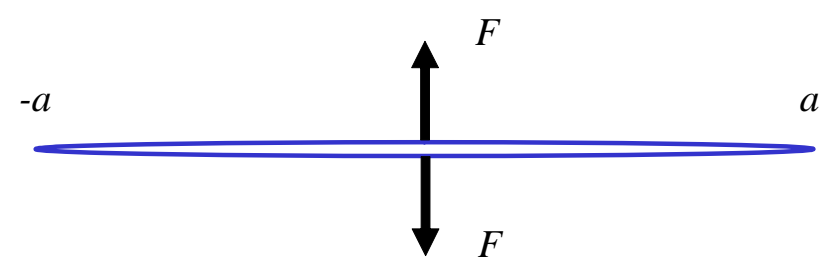

Fig. 3. Equivalent representation of the crack created by selfequilibrated stress fluctuations shown in Fig. 2. The force magnitude is given by the last equation in Eq. (10).

This result corresponds to the intuitive perception that on average, zero cause has zero effect. The results changes completely if the crack is created by the very stress fluctuations. So, suppose that the crack is initiated at the place where this realisation assumes a maximum and place the origin of the co-ordinate frame at that place. For a different realisation of random stress fluctuations the maximum stress will be at a different location, so the crack will also form at the new location. We always place the origin of the co-ordinate frame at the location where the crack is going to be formed.

Now, because the crack centre is correlated with the stress field, the mean value of the stress intensity factor will no longer vanish. If $\rho$ is the correlation length of the random stress fluctuations then the stress within this distance from the origin will survive averaging such that

$\bar{K}_{I} \sim \frac{1}{\sqrt{\pi a}} \int_{-\rho}^{\rho} \overline{\Delta \sigma_{y y}}(t) \sqrt{\frac{a+t}{a-t}} d t>0$

Here the stress at the origin is assumed to be positive.

More rigorous representation of the effect of stress fluctuations can be obtained by involving the concept of conditional average, as the stress present in Eq. (6) is, in fact, the conditional mathematical expectation, $E\left(\Delta \sigma_{y y}(t) \mid \Delta \sigma_{y y}(0)=\sigma_{t}\right)$, where $E$ stands for the operator of mathematical expectation and $\sigma_{t}$ is the local tensile strength of the material. Subsequently,

$$
\begin{aligned}
\bar{K}_{I} & =\frac{1}{\sqrt{\pi a}} \int_{-\infty}^{\infty} E\left(\Delta \sigma_{y y}(t) \mid \Delta \sigma_{y y}(0)=\sigma_{t}\right) \sqrt{\frac{a+t}{a-t}} d t \\
& \sim \frac{1}{\sqrt{\pi a}} \int_{-\rho}^{\rho} E\left(\Delta \sigma_{y y}(t) \mid \Delta \sigma_{y y}(0)=\sigma_{t}\right) \sqrt{\frac{a+t}{a-t}} d t
\end{aligned}
$$

For a particular case of Gaussian stress fluctuations the statistical distribution is controlled by unconditional mathematical expectation and the correlation function $B(t)$

$B(t)=E\left(\Delta \sigma_{y y}(0) \Delta \sigma_{y y}(t)\right)$.

Then the conditional mathematical expectation can be expressed in the following form (e.g., Feller, 1971)

$\overline{\sigma_{y y}}(t)=E\left(\Delta \sigma_{y y}(t) \mid \Delta \sigma_{y y}(0)=\sigma_{t}\right)=\sigma_{t} \frac{B(t)}{B(0)}$

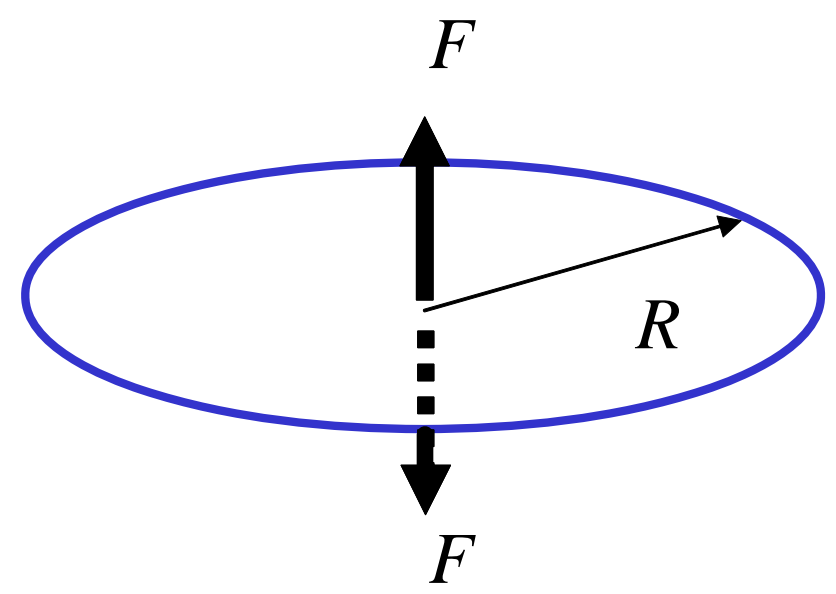

Fig. 4. Model of isotropic crack growth caused by stress fluctuations in 3-D - a disc-like crack of radius $R$ opened by a pair of concentrated forces.

Hereafter, for the sake of simplicity we return to our previous notations, which express the essence of the phenomenon without going into mathematical details. If the crack length is much higher than the correlation length then, obviously,

$\bar{K}_{I}=\frac{F}{\sqrt{\pi a}}, \quad F \sim \int_{-\rho}^{\rho} \overline{\sigma_{y y}}(t) \sqrt{\frac{a+t}{a-t}} d t \sim \int_{-\rho}^{\rho} \overline{\sigma_{y y}}(t) d t$,

$a \gg \rho$

This formula coincides with the expression for the stress intensity factor for a crack loaded with a pair of concentrated forces, Fig. 3.

One can observe that the stress intensity factor given by the first equation in Eq. (10) decreases with crack growth since the crack length enters denominator of the formula. The crack growth is therefore stable: in order to maintain crack growth one needs to increase the magnitude of the forces, that is the amplitude of the stress fluctuations. If the crack were loaded by stresses with not vanishing total force, the stress intensity factor, $K_{I} \cong \bar{\sigma}_{y y} \sqrt{\pi a}$ would increase as the crack grows, which indicates the unstable crack growth: in a material with uniform fracture toughness, once started the crack can grow indefinitely. It is interesting that while the stress fluctuations with zero average can support crack growth, the growth can only be stable: unstable crack propagation requires non-vanishing total force.

In the 3-D case the model of crack growth caused by stress fluctuations will be similar - a crack opened by a pair of concentrated forces. As further simplification necessary in 3-D, we assume that the crack grows isotropically as a disc-like crack, Fig. 4.

In this case, the mode I stress intensity factor for the disklike crack of radius $R$ has the form (e.g., Tada et al., 2000)

$K_{I}=\frac{F}{(\pi R)^{3 / 2}}$ 
This expression together with the criterion of crack growth

$K_{I}=K_{I c}$

will be used in the following two sections when the crack interaction and the formation of fracture patterns are discussed.

The local tensile strength, $\sigma_{t}$, plays a role of the first threshold: as soon as the amplitude of stress fluctuations reaches the local strength, a crack will be formed leading to fracture patterning. Its further development is also controlled by the fracture toughness of the material, which constitutes the second threshold in the multistage process of fracture pattern formation.

\section{Emergence of self-similar distributions}

We assume now that a number of cracks have been formed by the action of stress fluctuations and that they grow as disclike cracks each opened by a pair of concentrated forces of the same average magnitude $F$. We also assume that the material is isotropic and the cracks are situated at random locations and oriented chaotically such that the whole system remains isotropic.

As the cracks grow the effect of interaction between them becomes significant. In this case the interaction means that each crack is subjected to a combination of the pair of concentrated forces that open the crack and some additional, generally non-uniform stresses generated by all other cracks. This additional stress, due to the randomness in crack locations, has zero mean as it is not associated with any external loading. Subsequently, solving the problem of interaction means finding these additional stresses, which is computationally involved since it requires solving a system of integral equations of the order of $3 N$, where $N$ is the number of cracks.

A major simplification can be achieved if one takes into account that the neighbouring cracks cannot be of the same size, as the conditions and circumstances of their appearance were different. We will bring this notion to its extreme and assume that the cracks have a wide distribution of sizes (Salganik, 1973). This means that the cracks of close size are in low concentration and hence do not interact directly. The interaction is essential only between the cracks of markedly different sizes. If this is the case, then the method of effective characteristics can be applied to compute the average effect of interaction. According to the method (Salganik, 1973) each crack can be considered in an effective medium defined by all smaller cracks.

Figure 5 illustrates the idea. Firstly we replace the original material containing only the smallest cracks with an effective medium which provides the same average response as the replaced system. Here "average" is understood in terms of stress and strain averaged over volume elements that are much larger than the corresponding crack size (in this case the size of the smallest cracks) and contain sufficiently large
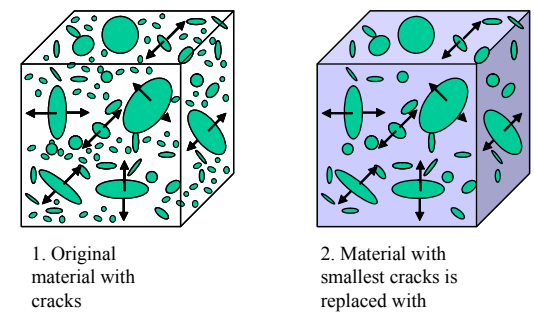

2. Material with

smallest cracks is

replaced with

effective medium

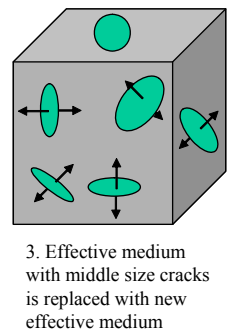

Fig. 5. Method of effective characteristics in modelling the crack interaction: 1. The original material with cracks which are opened by pairs of concentrated forces (not all of them are shown in the picture). 2 . The material with the smallest cracks is replaced by an effective medium with the same average response. 3 . The effective medium with middle size cracks is replaced with a new effective medium with the same average response.

number of these cracks. Because of elasticity these average stress and strain are related by Hooke's law with the parameters called effective characteristics. As we assumed that the neighbouring cracks (the ones that interact most strongly) had very large difference in sizes, we could further suppose that they were much larger than the size of the averaging volume elements. Subsequently, we can consider these larger cracks as situated in the effective medium. Thus the influence of the smaller cracks is accounted for in an integral fashion through the effective medium. The particular calculations are facilitated by the assumption that the cracks of similar size do not interact; hence approximation of low concentration based on the solution for a single crack in infinite medium can be used.

We can repeat this procedure with the cracks of the next size and so on, as shown in Fig. 5. At each step, when the properties of the corresponding effective medium are calculated the cracks of the relevant sizes are considered to be noninteracting allowing the use of the approximation of low concentration. The interaction is thus accounted for through the succession of the effective media with properties eventually determined by all smaller cracks. In essence this scheme is an asymptotic one: the accuracy of the approximation thus produced increases as the difference in sizes of interacting cracks increases and the concentration of the cracks of the same size decreases (see proof in Dyskin, 2002).

The outlined procedure dealt with the "upward" interaction, i.e. the average influence of small cracks on the large ones. The "downward" interaction, i.e. the influence of the additional stresses generated by large cracks on the small ones does not need to be considered if we are only interested in the average effects, as the average stress generated by any crack is zero (see Appendix for the proof).

We now use the above method to determine the size distribution the cracks driven by the concentrated forces assume due to interaction. We start with expressing the effective moduli for the material with cracks up to a given radius. 
Suppose the crack sizes are distributed according to a probability density function $f(R)$ with the usual normalisation:

$$
\int_{R_{0}}^{R_{\max }} f(R) d R=1
$$

Here $R_{0}, R_{\max }$ are the minimum and maximum crack sizes respectively. If $N$ is the total number of cracks per unit volume ( $N$ has units of inverse meter cubed), then the number of cracks per unit volume with radii between $R$ and $R+d R$ is

$N(R, R+d R)=N f(R) d R$

The total number of cracks is supposed to be constant, i.e. the generation of new cracks is neglected. We also use the dimensionless crack concentration

$d \mathrm{v}=N R^{3} f(R) d R, \quad \mathrm{v}(R)=N \int_{R_{0}}^{R} R^{3} f(R) d R$

Let the Young's modulus and Poisson's ratio of the effective medium replacing the material with cracks of radii up to $R$ be $E(R)$ and $v(R)$. Then, according to Salganik (1973), the effective characteristics for the medium with new cracks of radii between $R$ and $R+d R$ can be computed by considering these new cracks as being immersed in the effective medium whose properties are characterised by $E(R)$ and $v(R)$. These new cracks are considered to be non-interacting as their concentration is, according to the first equation in Eq. (15), infinitesimal. We use the approximation of low concentrations, which gives an expression linear with respect to $d \mathrm{v}$. The method described is called the "differential self-consistent method". For the case of isotropic distribution of disc-like cracks the effective characteristics have the form (e.g., Salganik, 1973)

$$
\begin{aligned}
& E(R+d R)=E(R)\left[1-\frac{16}{45}(10-3 v(R)) \frac{1-v(R)^{2}}{2-v(R)} d \mathrm{v}\right] \\
& v(R+d R)=v(R)-\frac{16}{15}(3-v(R)) \frac{1-v(R)^{2}}{2-v(R)} d \mathrm{v} \\
& E(0)=E_{0}, \quad v(0)=v_{0}
\end{aligned}
$$

Here $E_{0}, \nu_{0}$ are the material's Young's modulus and the Poisson's ratio respectively. The right hand sides of the equations represent the change in macroscopic moduli of the material associated with the presence of cracks of radius $R$. It is important to note that the effective characteristics are solely determined by the crack concentration rather than separately by their size and number. Also, one can observe that the effective Poisson's ratio decreases, as the crack concentration increases. This means that for the sake of simplicity the Poisson's ratio can be neglected, $v(R)=0$, which leads to the following simplified equation

$$
\frac{d E(R)}{E(R)}=-\frac{16}{9} N R^{3} f(R) d R, \quad E\left(R_{0}\right)=E_{0}
$$

As the cracks grow, their distribution undergoes evolution. The growth of the cracks is affected by the interaction and cracks of different size are affected differently. In order to account for this consider the condition of growth of a crack or radius $R$. In the spirit of above approximation, we shall consider this crack as being immersed in an effective medium with effective characteristics determined by all smaller cracks. As these smaller cracks make the material softer, as stipulated by the negative sign in formula (17), the opening of the crack in question will be larger as compared to the same crack in the original material. The crack opening is inversely proportional to the Young's modulus (as routinely followed from the dimension analysis) hence the increase in the average crack opening associated with the interaction is proportional to the factor $E_{0} / E(R)$, the influence of the Poisson's ratio being neglected.

Crack growth is controlled by stress concentration in a small vicinity of the crack contour. It is important to realise here that while the crack opening is on average dictated by the effective medium replacing the material with smaller cracks, the microscopic stress distribution at the crack contour is acting in the original material. Hence, the transition from the crack opening profile to the stress concentration is controlled by the Young's modulus of the original material. Therefore the increase in the stress concentration due to the interaction is proportional to the increase in the crack opening. Subsequently, the stress intensity factor controlling the stress concentration is, on average, increased proportionally to the ratio of the Young's modulus of the original material to the effective Young's modulus created by the cracks of radius $R$ in the original material ${ }^{1}$

$$
\frac{\left\langle K_{I}(R)\right\rangle}{K_{I}^{0}}=\frac{E_{0}}{E(R)}
$$

Using Eq. (18) in conjunction with the expression for the stress intensity factor (Eq. 11) for the disc-like crack opened by a pair of concentrated forces in the original material and the criterion of crack growth (Eq. 12) one obtains

$$
E(R)=\frac{F E_{0}}{K_{I c}(\pi R)^{3 / 2}}
$$

Differentiating Eq. (19) with respect to $R$ and substituting into Eq. (17) gives

$f(R)=\frac{27}{32 R^{4}}$

What is obtained is a power law. It is not yet self-similar distribution as it has boundaries lower and upper cutoffs, $R_{0}, R_{\max }$. The lower cutoff, i.e. the average radius of the crack not affected by the interaction, can be determined from Eqs. (11) and (12):

$$
R_{0}=\frac{F^{2 / 3}}{\pi K_{I c}^{2 / 3}}
$$

\footnotetext{
${ }^{1}$ A rigorous description and proof of this concept could be found in Dyskin (2002).
} 
The upper cutoff is dictated by the normalisation condition (13):

$$
\frac{R_{\max }}{R_{0}}=\left[1-\left(\frac{F}{F_{\max }}\right)^{2}\right]^{-1 / 3}, \quad F_{\max }=\frac{3 \pi^{3 / 2} K_{I c}}{\sqrt{2 N}}
$$

If the concentrated force i.e. the loading associated with the action of the stress fluctuations increases, the upper cutoff increases and becomes infinite as the concentrated force $F$ approaches a critical value, $F_{\max }$. This indicates that with the increase in the loading parameter (the amplitude of stress fluctuations in our case) the crack distribution approaches the self-similar one. Subsequently, the critical value, $F_{\max }$ or the corresponding amplitude of the stress fluctuations can be considered as a threshold after which the fracture pattern becomes self-similar. This constitutes the third threshold in the multistage process of fracture pattern formation.

We can conclude that in the simple case of isotropically oriented cracks the interaction constitute a mechanism of the emergence of self-similarity in the crack distribution. The isotropic crack distribution, albeit important, is only one example of distributions observed in reality. Another important class is given by systems of parallel cracks. Unfortunately, the direct analysis of the sort presented above meats with considerable technical difficulties. These difficulties could be partially bypassed if we consider self-similar distributions from the very beginning and check whether the crack growth preserves self-similarity. Preservation of self-similarity is obviously a necessary condition of emergence of self-similar distributions and it is easier to check. The corresponding formalism is introduced in the following section.

\section{Mechanics of self-similar materials}

The approach to modelling crack interaction described in the previous section - the introduction of a set of effective media that model the original material with cracks of size smaller than the specified one - is especially attractive in the case of self-similar crack distributions. Indeed, the transition from one size to another utilised in the method directly corresponds to the multi-scale nature of self-similar distributions. Furthermore, additional simplification is delivered by the case of self-similarity, since it implies that all quantities are expressed by power functions of scale (e.g., Barenblatt and Botvina, 1980; Gelikman and Pisarenko, 1989; Zosimov and Lyamishev, 1995). Subsequently, in the isotropic case

$E(R) \sim R^{-\alpha}, \quad v=\mathrm{const}$

Here the scaling exponent for the Young's modulus $\alpha>0$, while the scaling exponent for the Poisson's ratio vanishes since the Poisson's ratio is bounded: $-1 \leq v \leq 0.5$.

The complication seem to come when the material with cracks is anisotropic (e.g., the cracks are arranged in one or several sets of parallel cracks), the Hooke's law is represented by a fourth rank tensor of elastic moduli, $C_{i j k l}, \quad i, j, k, l=1 . .3$ or the inverse tensor of elastic compliances, $A_{i j k l}, i, j, k, l=1 . .3$, each having 21 independent components. One would expect that then the scaling is controlled by 21 exponents. The surprising result is that the scaling remains simple: Dyskin (2004) proved that all non-zero components of a tensor must scale with the same exponent. In particular,

$C_{i j k l}(H)=c_{i j k l} H^{\alpha}, \quad A_{i j k l}(H)=a_{i j k l} H^{\beta}$,

$i, j, k, l=1 . .3$

Thus scaling is always isotropic, only the pre-factors, $c_{i j k l}$ and $a_{i j k l}$ describe anisotropy.

We can now use this scaling and preform transition from scale $R$ to $R+d R$ in the spirit of Eq. (16):

$d C_{i j k l}(R)=-\Delta C_{i j k l}(R) d R$

where $-\Delta C_{i j k l}(R) d R$ is the contribution of cracks of radius $R$ into the effective moduli. Because of self-similarity and the fact that the scaling is controlled by a single exponent

$\Delta C_{i j k l}(R)=\Delta c_{i j k l} R^{\kappa}$

Substituting Eqs. (24), (26) into Eq. (25) one obtains that $\kappa=\alpha-1$ and

$\alpha c_{i j k l}=-\Delta c_{i j k l}$

This is a system of, generally, 21 equations for 22 unknowns. Since the pre-factors for both moduli and the increments have the same units, one of the pre-factors can be chosen arbitrarily, while the others and the exponent can be found from Eq. (27). We also note that this system is a general one; no assumptions on the nature of interaction between the cracks have yet been made.

Now, in order to solve system (27) further analysis of selfsimilar crack distribution is needed. We first note that the general form of self-similar distributions is

$f(R)=\frac{w}{R^{m}}$

The main difficulty with such a distribution is that because it ranges from zero to infinity, the usual normalisation

$$
\int_{0}^{\infty} f(R) d R=1
$$

involves divergent integral for any $m$. In order to overcome this difficulty, we view self-similar distributions only as approximations of the real ones which range between lower and upper cut-offs, Dyskin (2004). Subsequently, the normalisation factor should depend on the cut-offs. We will determine it in such a way that the self-simular distribution produces 
the same total concentration, $\mathrm{v}_{t}$, of cracks between the lower and upper cut-offs, as in the real system. Then

$$
N \int_{R_{0}}^{R_{\max }} R^{3} f(R) d R=v_{t}
$$

Using Eq. (28) one obtains

$$
\mathrm{v}_{t}=\left\{\begin{array}{c}
\frac{N w}{4-m} R_{\max }^{4-m}\left[1-\left(\frac{R_{0}}{R_{\max }}\right)^{4-m}\right] \text { when } \quad m \neq 4 \\
N w \ln \frac{R_{\max }}{R_{0}} \quad \text { when } m=4
\end{array}\right.
$$

It is obvious from Eq. (31) that in order to keep the total concentration constant, the concentration factor, $w$, must tend to zero as the range of self-similarity widens, $R_{\max } / R_{0} \rightarrow \infty$. In this sense, the self-similarity can be regarded as an asymptotic property.

In order to be able to use the method of effective characteristics considered above, we need the concentration of cracks of similar size to be infinitesimal. Consider the concentration of cracks in the range $(R / n, R)$, where $n>1$ :

$\mathrm{v}\left(\frac{R}{n}, R\right)=N \int_{\frac{R}{n}}^{R} R^{3} f(R) d R=\left\{\begin{array}{l}\frac{N w}{4-m} R^{4-m}\left(1-n^{m-4}\right) \\ \text { when } m \nRightarrow 4 \\ N w \ln n \\ \text { when } m=4\end{array}\right.$

Obviously, the crack concentration is independent of the crack size only when $m=4$. Furthermore, as follows from Eq. (31) only when $m=4$, the concentration of cracks of each size tends to zero uniformly with respect to the crack size as the range of self-similarity widens, $R_{\max } / R_{0} \rightarrow \infty$ :

$\mathrm{v}\left(\frac{R}{n}, R\right)=\mathrm{v}_{t} \frac{\ln n}{\ln \left(R_{0} / R_{\max }\right)} \underset{R_{0} / R_{\max } \rightarrow \infty}{\longrightarrow} 0$

Dyskin (2004) called the case $m=4$ the "self-similarity in narrow sense". In this case the probability to find in a vicinity of a crack of size $R$ other cracks of similar size also tends to zero as $R_{\max } / R_{0} \rightarrow \infty$, which justifies the use of the differential self-consistent method. It is not surprising then that this type of self-similarity is reproduced by the mechanism described in the previous section.

Opposite to this, when, say, $m<4$

$\mathrm{v}\left(\frac{R}{n}, R\right)=\mathrm{v}_{t}\left(\frac{R}{R_{\max }}\right)^{4-m}\left(1-n^{m-4}\right)$

This concentration tends to zero as $R_{\max } / R_{0} \rightarrow \infty$ for any fixed crack size $R$, but does not tend to zero for $R \sim R_{\max }$. What this means is that asymptotically the main crack concentration resides with the largest cracks, while the total concentration of all smaller cracks is negligible. Similarly, for $m>4$ the smallest cracks are in concentration close to $v_{t}$, while the concentration of all other (larger) cracks is vanishing.
Now, using the crack distribution (28) with $m=4$ and the concentration factor $w$ determined by the corresponding equation from Eq. (31) we can rewrite the general equation (27). According to the differential self-consistent method, the pre-factor of the moduli increment $\Delta c_{i j k l}$ is determined by the contribution of non-interacting inhomogeneities considered in an effective continuum. This contribution is proportional to the concentration of the group of inhomogeneities at hand, and hence to $w$ since the inhomogeneities of the same scale do not interact due to their low concentration. System (27) can then be rewritten in the form

$\alpha c_{i j k l}=-w \Lambda_{i j k l}\left(c_{1111}, c_{1112}, \ldots c_{3333}\right), \quad i, j, k, l=1 . .3(35)$

where the function $\Lambda_{i j k l}$, homogeneous of the first degree, represents the contribution of cracks to the elastic moduli at each step of the self-consistent method.

A similar system could be obtained for the pre-factors for the scaling of the tensor of effective compliances, Dyskin (2004):

$\beta a_{i j k l}=w S_{i j k l}\left(a_{1111}, a_{1112}, \ldots a_{3333}\right), \quad i, j, k, l=1 . .3$

Here the function $S_{i j k l}$ also homogeneous of the first degree, represents the contribution of cracks to the compliances at each step of the self-consistent method.

For the case of isotropic distribution of disc-like cracks considered in the previous section, function $\Lambda_{i j k l}$ can be extracted from Eq. (16). As a result, system (35) assumes the form

$\left\{\begin{array}{l}\alpha e=-\frac{16}{45}(10-3 v) \frac{1-v^{2}}{2-v} w e \\ \alpha v=-\frac{16}{15}(3-v) \frac{1-v^{2}}{2-v} w v\end{array}\right.$

where $e$ is a pre-factor for the scaling of the Young's modulus.

This system has only one non-trivial solution:

$v=0, \quad E=e R^{-\frac{16}{9} w}$

Consider now the case of a set of parallel disc-like cracks. Suppose the cracks are oriented perpendicular to the $x_{3}$ axis and distributed self-similarly with the distribution function (28) and $m=4$. For this case the effective compliances can be found from the general solution by Vavakin and Salganik (1978) for a transverse-isotropic material with disc-like cracks parallel to the plane of isotropy. The Hooke's law for a transverse isotropic material is expressed in the co-ordinate set $\left(x_{1}, x_{2}, x_{3}\right)$ through the compliances in a $6 \times 6$ matrix form as follows

$\left\{\begin{array}{l}\varepsilon_{11}=A_{11} \sigma_{11}+A_{12} \sigma_{22}+A_{13} \sigma_{33} \\ \varepsilon_{22}=A_{12} \sigma_{11}+A_{11} \sigma_{22}+A_{13} \sigma_{33} \\ \varepsilon_{33}=A_{13} \sigma_{11}+A_{13} \sigma_{22}+A_{33} \sigma_{33} \\ \varepsilon_{23}=\frac{1}{2} A_{44} \sigma_{23} \\ \varepsilon_{13}=\frac{1}{2} A_{44} \sigma_{13} \\ \varepsilon_{12}=\left(A_{11}-A_{22}\right) \sigma_{12}\end{array}\right.$ 
Vavakin and Salganik's (1978) solution gives in the case of low crack concentration:

$$
\left\{\begin{aligned}
A_{11}= & A_{11}^{0}, \quad A_{12}=A_{12}^{0}, \quad A_{13}=A_{13}^{0} \\
A_{33}= & A_{33}^{0}+\frac{8 v}{3 A_{11}^{0}} \sqrt{B^{0}\left[A_{11}^{0} A_{33}^{0}-\left(A_{13}^{0}\right)^{2}\right]} \\
A_{44}= & A_{44}^{0}+\frac{16 v}{3} A_{44}^{0} \frac{\sqrt{B^{0}\left[\left(A_{11}^{0}\right)^{2}-\left(A_{12}^{0}\right)^{2}\right]}}{A_{11}^{0} A_{44}^{0}+\sqrt{\frac{1}{2} B^{0} A_{44}^{0}\left[A_{11}^{0}+A_{12}^{0}\right]}} \\
B^{0}= & A_{11}^{0} A_{44}^{0}+2 A_{13}^{0}\left[A_{11}^{0}-A_{12}^{0}\right] \\
& +2 \sqrt{\left[A_{11}^{0} A_{33}^{0}-\left(A_{13}^{0}\right)^{2}\right]\left[\left(A_{11}^{0}\right)^{2}-\left(A_{12}^{0}\right)^{2}\right]}
\end{aligned}\right.
$$

Here $A_{11}^{0}, A_{12}^{0}, A_{13}^{0}, A_{33}^{0}, A_{44}^{0}$ are the compliances of the material.

The scaling equations (36) after rewriting in the $6 \times 6$ matrix form can be obtained by replacing $v$ with $w$ bringing $A_{i i}^{0}$ to the left-hand sides and then replacing $A_{i i}-A_{i i}^{0}$ with $\beta a_{i i}$ and, finally, replacing $A_{i i}^{0}$ with $a_{i i}$ in the remaining parts. This yields the following scaling equations

$$
\left\{\begin{aligned}
\beta a_{11} & =0, \quad \beta a_{12}=0, \quad \beta a_{13}=0 \\
\beta a_{33} & =\frac{8 w}{3 a_{11}} \sqrt{B\left[a_{11} a_{33}-\left(a_{13}\right)^{2}\right]} \\
\beta & =\frac{16 w}{3} \frac{\sqrt{B\left[\left(a_{11}\right)^{2}-\left(a_{12}\right)^{2}\right]}}{a_{11} a_{44}+\sqrt{\frac{1}{2} B a_{44}\left[a_{11}+a_{12}\right]}} \\
B & =a_{11} a_{44}+2 a_{13}\left[a_{11}-a_{12}\right] \\
& +2 \sqrt{\left[a_{11} a_{33}-\left(a_{13}\right)^{2}\right]\left[\left(a_{11}\right)^{2}-\left(a_{12}\right)^{2}\right]}
\end{aligned}\right.
$$

Suppose $\beta \neq 0$, then the first three equations of Eq. (41) produce $a_{11}=a_{12}=a_{13}=0$. On the other hand, the third equation produces $\beta=0$. This contradiction means that the scaling exponent vanishes. As a result we obtain the following scaling law:

$A_{i j}=a_{i j} H^{\beta}, \quad \beta=0 \Rightarrow A_{i j}=a_{i j}$

This means that the compliances and hence the moduli are scale-invariant.

\section{Stability of self-similar crack distributions}

We now use the introduced concepts to investigate the stability of two self-similar crack distributions: isotropically oriented cracks and a set of parallel cracks.

Firstly we need to determine the scaling for the average stress intensity factor. In line with Eq. (18), the average SIF scales as

$$
\left\langle K_{I}(R)\right\rangle \sim R^{-\alpha}
$$

For isotropic cack distribution this expression is a straightforward generalisation of Eq. (18). It is however valid for any anisotropy since all non-zero components of the tensor of elastic moduli scale with the same exponent $\alpha$ (the scaling law is always isotropic).
We now substitute scaling (43) into the expression for the stress intensity factor (Eq. 11) and obtain

$$
\left\langle K_{I}(R)\right\rangle \sim R^{-\alpha-\frac{3}{2}}
$$

When scaling (44) is substituted into the criterion of crack growth (12) under the assumption that the latter is scaleinvariant it follows that the exponent in Eq. (44) must be zero. Therefore, if the cracks are to grow keeping self-similarity, the exponent of the moduli scaling cannot be arbitrary, but should satisfy the following condition

$\alpha=-\frac{3}{2}$

For isotropic distribution of disc-like cracks this, according to scaling law (38), corresponds to

$w=\frac{27}{32}$

Thus, the form of the crack distribution remains the same. This means that in order to maintain the concentration factor constant the crack growth must only affect the lower and upper cutoffs of the distribution: both $R_{\max }$ and $R_{0}$ get increased as dictated by the normalisation equation (30).

After substituting Eq. (46) into Eq. (28) the distribution (20) is recovered, which indicates that the analysis made on the basis of self-similar mechanics is consistent with the model of the emergence of self-similar distribution of isotropically oriented cracks.

In the case of a single set of parallel cracks condition (46) cannot be satisfied due to the trivial scaling $(\alpha=0)$. Therefore, for such crack distributions, the self-similarity cannot be maintained: it will be destroyed by crack growth. Thus the necessary condition of emergence of self-similar distribution is not met, which means that the mechanism of stress fluctuations and crack interaction cannot produce a single set of parallel cracks with self-similar distribution of sizes.

\section{Permeability in the presence of self-similar crack dis- tributions}

We now consider the change in permeability, $K$, the selfsimilar crack distribution induces in an already permeable material. This is a special case when the cracks enhance permeability because each of them serves as a conduit in a part of the material it belongs to without forming interconnected fracture networks. Naturally, the self-similar nature of the crack distribution will lead to a power law scaling of the effective permeability:

$K=k R^{\eta}$

where $\eta$ is the scaling exponent and $k$ is a pre-factor. Hereafter, we only consider the case of isotropically oriented cracks. 
We determine the scaling law for the effective permeability, in the spirit of the approach described in Sect. 2. For that we need the expression for the effective permeability of a material with low concentration of cracks. In order to use an existing solution we use the well known analogy between the phenomena of permeability and thermal conductivity. The full analogue will be effective thermal conductivity, $\lambda$, of a material with isotropically oriented thin ellipsoidal inclusions of a material with extreme thermal conductivity. The corresponding solution was developed by Salganik (1974). According to this solution, the effective permeability reads

$\lambda=\lambda_{0}\left(1+\frac{32}{9} \mathrm{v}\right)$

Here $\lambda_{0}$ is the thermal conductivity of the material, $\mathrm{v} \ll 1$ is the crack concentration. As the cracks are assumed absolutely conductive, their presence increases permeability. The effective permeability of a material with cracks of concentration $\mathrm{v}$ can formally be obtained from Eq. (48) by replacing thermal conductivity $\lambda$ with permeability $K$.

$K=K_{0}\left(1+\frac{32}{9} \mathrm{v}\right)$

where $K_{0}$ is the permeability of the material.

Analogously to Eq. (35), for self-similar crack distribution $f(R)=w R^{-4}$ the scaling equation for permeability reads

$\eta k=\frac{32}{9} w k$

From here we immediately have

$\eta=\frac{32}{9} w$

In the case when the self-similar crack distribution emerged as a result of crack growth induced by stress fluctuations, $w=27 / 32$ and we finally have

$K=k R^{3}$

This scaling law, if detected for a particular fracture system could serve as an indicator that these cracks were created by the mechanism described in this paper.

\section{Conclusions}

Fluid flow through geomaterials is strongly affected by the presence of fractures. Fracture systems result from complex non-linear processes of crack initiation and growth. The fracture pattern formation is a multiscale process controlled by a number of thresholds. As the phenomenon of fracturing is a highly non-linear process, the spatial stress fluctuations - stress non-uniformity associated with heterogeneity of the material or with the presence of residual stresses - can considerable affect the crack formation even if the mean value of the stress fluctuations is zero.
Fracture pattern formation driven by stress fluctuations is a multistage process. At the first stage, microcracks are generated at the places subjected to the highest tensile stresses when the amplitude of stress fluctuations exceeds the first threshold - local tensile strength of the material. These microcracks can grow to a scale macroscopic with respect to the correlation length of the stress fluctuation because, albeit the average stress is zero, the conditional average stress (under the condition that at the place of microcrack initiation the stress exceeds the tensile strength) is positive. This constitutes the second stage of fracture pattern formation. In order to ensure crack growth the amplitude of stress fluctuations should be sufficient to induce the stress concentrations at the crack contour necessary to maintain its growth, i.e. to make the stress intensity factor (a coefficient at the stress singularity) exceed the fracture toughness. This introduces the second threshold. Further crack growth is supported by interaction with other cracks. The effect of interaction can be accounted for by considering each crack as being immersed in an effective continuum determined by all cracks of smaller sizes (the differential self-consistent scheme of the method of effective characteristics). For isotropic crack orientations, this mechanism produces self-similar distribution of crack sizes, which constitutes the third stage of the process of fracture pattern formation. This is achieved when the amplitude of stress fluctuations reached a certain value at which the maximum crack size becomes infinite. This constitutes the third threshold.

For materials with self-similar crack distribution the scaling of elastic moduli and the average stress intensity factors are governed by the power laws. Furthermore it can be shown that scaling of tensors is always isotropic: all nonzero components of a tensor scale with the same exponent. The anisotropy affects only pre-factors.

The analysis of the stability of self-similar crack distributions with respect to crack growth shows that in the case of isotropic crack orientations the self-similar distribution is stable, while the self-similar distribution of parallel cracks is not. Therefore, the latter cannot be produced as a result of the action of stress fluctuations and crack interaction.

Permeability of a material with self-similar distribution of isotropically oriented cracks, which do not yet form connected networks, is also governed by a power scaling law. For the crack distribution produced by the action of stress fluctuations the permeability increases as cube of crack radius. This property could be used for detecting this specific mechanism of formation of self-similar crack distributions. 


\section{Appendix A}

\section{The proof that the average stress generated by a crack is zero}

Consider a crack with internal surface $S_{0}$ occupying a volume $V$ with external surface $S$, which is unconstrained and free of load. Suppose in response to tractions $n_{i} \sigma_{i j}^{0}$, where $n_{i}$ is a normal vector to the crack surface directed inwards, the crack produces stress field $\sigma_{i j}(\boldsymbol{x}), \boldsymbol{x} \in V$. We also assume that there are no body forces acting on the volume. Consider the average stress field

$$
\left\langle\sigma_{i j}\right\rangle=\frac{1}{V} \int_{V} \sigma_{i j}(\boldsymbol{x}) d V \boldsymbol{x}
$$

We use the following identity which is based on the Gauss' theorem

$$
\begin{aligned}
\int_{S+S_{0}} \sigma_{i k}(\boldsymbol{x}) x_{j} n_{k} d S= & \int_{V}\left(\sigma_{i k}(\boldsymbol{x}) x_{j}\right)_{, k} d V \boldsymbol{x} \\
= & \int_{V} \sigma_{i k, k}(\boldsymbol{x}) x_{j} d V \boldsymbol{x} \\
& +\int_{V} \sigma_{i k}(\boldsymbol{x}) \delta_{j k} d V \boldsymbol{x}
\end{aligned}
$$

The first integral in the right-hand part is equal to zero because of the equations of equilibrium (the body forces are zero by assumption). The second integral is equal to the average stress times V. Furthermore, since the outer boundary of the volume is free from load

$$
\left\langle\sigma_{i j}\right\rangle=\frac{1}{V} \int_{V} \sigma_{i j}(\boldsymbol{x}) d V \boldsymbol{x}=\frac{1}{V} \int_{S_{0}} \sigma_{i k}(\boldsymbol{x}) x_{j} n_{k} d S=0 \text {.(A3) }
$$

The last integral is equal to zero because the tractions acting on the crack faces are in equilibrium and the gap between faces (crack opening) is zero in the elastic formulation.

Due to ergodicity, one can obtain that

$\bar{\sigma}_{i j}=\left\langle\sigma_{i j}\right\rangle=0$

Acknowledgements. The author acknowledges the financial support through the Australian Research Council Discovery Grant (DP0559737) and the financial support by the Australian Computational Earth Systems Simulator - Major National Research Facility (ACcESS MNRF).

Edited by: M. Sivapalan

\section{References}

Bak, P., Tang, C., and Wiesenfeld, K.: Self-organised criticality. An explanation of 1/f noise, Phys. Rev. Lett., 59, 381-394, 1987.

Barenblatt, G. I. and Botvina, L. R.: Application of the similarity method to damage calculation and fatigue crack growth studies. Defects and Fracture, edited by: Sih, G. C. and Zorski, H., Martinus Nijhoff Publishers, 71-79, 1980.

Barton, C. A. and Zoback, M. D.: Self-similar distribution and properties of macroscopic fractures at depth in crystalline rock in the Cajon Pass scientific drill hole, J. Geophys. Res., 97B(4), 5181-5200, 1992.

Baxter, G. W., Behringer, R. P., Fagert, T., and Johnson, G. A.: Pattern formation in flowing sand, Phys. Rev. Lett. 62, 28252828, 1989.

Dubois, J.: Non-Linear Dynamics in Geophysics. John Wiley and Sons, Chichester, New York, Weinheim, Brisbane, Singapore, Toronto, 1998.

Dyskin, A. V.: On the role of stress fluctuations in brittle fracture, Intern. J. Fracture, 100, 29-53, 1999.

Dyskin, A. V.: Self-similar crack patterns induced by spatial stress fluctuations, Fatigue and Fracture of Engineering Materials and Structures, 25, 187-200, 2002.

Dyskin, A. V.: Effective characteristics and stress concentrations in materials with self-similar microstructure, Int. J. Solids Struct., 42(2), 477-502, 2004.

Feller, W.: An Introduction to Probability Theory and its Applications, vol. II, John Wiley \& Sons, Inc. New-York, London, Sydney, Toronto, 1971.

Gelikman, M. B. and Pisarenko, V. F.: About the self-similarity in geophysical phenomena, in: Discrete Properties of the Geophysical Medium, Nauka, Moscow (in Russian), 109-130, 1989.

Gillespie, P. A., Howard, C. B., Walsh, J. J., and Watterson, J.: Measurement and characterisation of spatial distribution of fractures, Tectonophysics, 226, 113-141, 1993.

Haken, H.: Synergetics, Springer, Berlin New York, 1978.

Hodkiewicz, P. F., Weinberg, R. F., Gardoll, S. J., and Groves, D. I.: Complexity gradients in the Yilgarn Craton: fundamental controls on crustal-scale fluid flow and the formation of world-class orogenic-gold deposits, Australian J. Earth Sci., 52, 831-841, 2005.

Langer, J. S.: Instabilities and pattern formation in crystal growth, Rev. Modern Phys., 52(1), 1-28, 1980.

Langer, J. S.: Dendrites, viscous fingers, and the theory of pattern formation, Science, 243, 1150-1156, 1989.

Olding, N. E.: Network properties of a two-dimensional nature fracture pattern, PAGEOPH, 138, 95-114, 1992.

Salganik, R. L.: Mechanics of bodies with many cracks, Mech. of Solids, 8, 135-143, 1973.

Salganik, R. L.: Transport processes in bodies with many cracks, Eng. Phys. J. (in Russian), XXVII, No. 6, 1069-1075, 1974.

Scholz, C. H.: The Mechanics of Earthquakes and Faulting, Cambridge University Press, Cambridge, New York, Port Chester, Melbourne, Sydney, 1990.

Scholz, C. H. and Aviles, C.: The fractal geometry of faults and folding, in: Earthquake Source Mechanics, edited by: Das, S., Boatwright, J., and Scholz, C. H., Monograph series, 37, Washington, D.C., American Geophysical Union, 147-155, 1986.

Sornette, D.: Critical Phenomena in Natural Sciences, SpringerVerlag, Berlin, Heidelberg, New York, 2000. 
Stauffer, D. and Aharony, A.: Introduction to Percolation Theory, London, Taylor and Francis, 1992.

Tada, H., Paris, P. C., and Irwin, G. R.: The stress analysis of cracks. Handbook, Third edition, New York, ASME Press, 1985.

Turcotte, D. L.: Fractals and Chaos in Geology and Geophysics, Cambridge University Press, 1993.

Vavakin, A. S. and Salganik, R. L.: Effective elastic characteristics of bodies with isolated cracks, cavities, and rigid nonhomogeneities, Mech. of Solids, 13(2), 87-97, 1978.

Walgraef, D.: Spatio-Temporal Pattern Formation with Examples from Physics, Chemistry, and Materials Science, Springer, 1997.
Weiss, J. and Marsan, D.: Three-dimensional mapping of dislocation avalanches: clustering and space/time coupling, Science, 299, 89-92, 2003.

Yamamoto, H., Kojima, K., and Tosaka, H.: Fractal clustering of rock fractures and its modelling using cascade process, in: Scale Effects in Rock Masses, edited by: Pinto da Cunha, A., Balkema, Rotterdam, 93, 81-86, 1993.

Zosimov, V. V. and Lyamishev, L. M.: Fractals in wave processes, Physics-Uspekhi, 38(4), 347-384, 1995. 